\title{
Contacts of healthcare workers, patients and visitors in general wards in Singapore
}

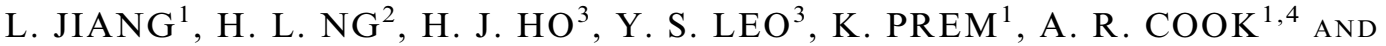 \\ M. I. $\mathrm{CHEN}^{1,3 *}$ \\ ${ }^{1}$ Saw Swee Hock School of Public Health, National University of Singapore and National University Health \\ System, Singapore, Singapore \\ ${ }_{2}^{2}$ Nursing Service, Tan Tock Seng Hospital, Singapore, Singapore \\ ${ }^{3}$ Institute of Infectious Diseases and Epidemiology, Tan Tock Seng Hospital, Singapore, Singapore \\ ${ }^{4}$ Yale-NUS College, National University of Singapore, Singapore, Singapore
}

Received 24 April 2017; Final revision 20 July 2017; Accepted 16 August 2017; first published online 8 September 2017

\section{SUMMARY}

To characterize contacts in general wards, a prospective survey of healthcare workers (HCWs), patients and visitors was conducted using self-reported diary, direct observation and telephone interviews. Nurses, doctors and assorted HCWs reported a median of 14, 18 and 15 contact persons over one work shift, respectively. Within $1 \mathrm{~h}$, we observed 3.5 episodes with $25.6 \mathrm{~min}$ of cumulative contact time for nurses, 2.9 episodes and $22 \cdot 1 \mathrm{~min}$ for doctors and 5.0 episodes with $44.3 \mathrm{~min}$ for assorted-HCWs. In interactions with patients, nurses had multiple brief episodes of contact; doctors had fewer episodes and less cumulative contact time; assorted-HCWs had fewer contact episodes of longer durations (than for nurses and doctors). Assortative mixing occurred amongst HCWs: those of the same HCW type were the next most frequent class of contact after patients. Over 24-h, patients contacted 14 persons with 23 episodes and $314.5 \mathrm{~min}$ of contact time. Patient-to-patient contact episodes were rare, but a maximum of five were documented from one patient participant. $22.9 \%$ of visitors reported contact with patients other than the one they visited. Our study revealed differences in the characteristics of contacts among different HCW types and potential transmission routes from patients to others within the ward environment.

Key words: Contact diary, direct observation, hospital ward, telephone interview.

\section{INTRODUCTION}

Healthcare workers (HCWs) have close interactions with patients and other contacts within the hospital [1]. Moreover, patients are at high risk for the acquisition and transmission of infectious diseases ranging from

\footnotetext{
* Corresponding author: M. I. Chen, Saw Swee Hock School of Public Health, National University of Singapore and National University Health System, 12 Science Drive 2, Kent Ridge, 117549, Singapore.

(Email: ephcicm@nus.edu.sg)
}

respiratory infections like influenza and Severe Acute Respiratory Syndrome (SARS) [2, 3] to contact transmissible drug resistant organisms such as Methicillinresistant Staphylococcus aureus (MRSA) [4].

Contacts between HCWs, the hospital environment, patients and visitors influence pathways for transmission and determine what interventions may be effective [5]. The characteristics of such contacts have been investigated through self-reported diaries, direct observation, and proximity-sensing technologies such as radiofrequency identification (RFID) 
systems [1, 6-8]. Of late, proximity-sensing technologies have been more commonly applied and are arguably less subject to observer and desirability bias and have less manpower requirements than diaries and direct observation. However, such technologies have their limitations; for instance, RFID would miss contacts not pre-tagged with the system [9] and cannot provide data on physical touch [10], which is critical for the transmission of some pathogens.

We used self-reported diaries and direct observation to investigate various characteristics of the contacts between HCWs, inpatients and visitors in a tertiary hospital ward. We also derive parameters for contact characteristics between HCWs, patients and visitors in the inpatient ward environment, details of which would be relevant to modelling disease transmission in this setting.

\section{METHODS}

\section{Study design and data collection}

We conducted a prospective study [11] of contacts where consenting participants knew in advance that they should report, or be under observation, regarding their contacts in general wards of Tan Tock Seng Hospital (TTSH) from June to December, 2013. TTSH is a 1400-bed acute care general hospital with a comprehensive suite of tertiary care specialties for adults, with the exception of obstetrics and gynaecological services. Our study was conducted on nine non-single-room wards and two single-room wards (averaging 38 and 16 beds respectively).

Four types of participants were recruited: Nurses (ward-based nurses, which included registered nurses, assistant nurses and nurse aides), doctors and assorted HCWs, patients and visitors. Assorted HCWs included a diversity of healthcare staff such as therapists, pharmacists and social workers; these were combined due to limited numbers in each of these sub-categories and also because they shared certain features, such as not being based in the ward environment for an entire workday and not working shifts within targeted wards. Nurses were recruited during their routine daily team meeting while other participant types were recruited individually when visiting, staying in, or working in the wards. We conducted the study across all days of the week including weekends. For HCWs, study team members specified the survey dates and work shifts to ensure different days and shifts (morning, afternoon and night) were represented proportionally to the scheduling of shifts within a typical ward environment. For patients, surveys covered the $24 \mathrm{~h}$ period starting from the point of recruitment (to minimize the chance they would be discharged before completing the study), while visitors completed their survey during the next working day after their hospital visit. Ethics approval was obtained from the National Healthcare Group (Singapore) Domain Specific Review Board, in accordance with relevant guidelines and regulations. Written informed consent was provided by each study participant.

A 'contact episode' [12] was defined as either a twoway conversation with three or more words in the presence of another person, or physical touch (for example a handshake, hug, or kiss) with another person, no matter whether the contact occurred between two persons or a group of people; contact episodes with physical touch were defined as 'physical contacts'. Individuals whom our participants had contact with are henceforth referred to as 'contact persons', with a participant potentially having multiple contact episodes with the same contact person within the observation period. Contact persons were grouped into six 'contact classes': doctors, nurses, assorted HCWs, patients, visitors (including family members) and others (not categorized into the previous classes).

Self-reported diaries and direct observation were used for HCWs and patients, who could choose to participate through either or both methods; for visitors, a telephone interview was used (Table 1). The scheduled timeframe of the survey for different participant types were: $24 \mathrm{~h}$ for patients, one work shift for HCWs (typically 8 and $10 \mathrm{~h}$ for day and night shifts, respectively) and one hospital trip for visitors (covering the entire period when he/she was in the hospital). However, for doctors and assorted HCWs, exposure to a specific inpatient ward varied substantially by the category of staff involved; their self-reported diaries included contact persons for the entire work shift, but direct observation covered only the period they were in a specific ward (between 2 and $8 \mathrm{~h}$ for participants in our study).

Self-reported diary: This was organized as a table where participants recorded features of their contact persons during the assigned day or shift: age (or approximate age range), gender, class of the contact person and whether they ever had physical contact with that person. Participants were instructed to use only one row for each contact person.

Direct observation: A team of trained observers were assigned to follow one specific participant for a designated period. After each contact episode had occurred, observers recorded the features of the 
Table 1. Overview of survey method for different types of participants

\begin{tabular}{llll}
\hline \hline Type of participant & Survey method(s) & Period assessed & Type of data collected \\
\hline Nurses & Direct observation & One work shift & Contact episodes \\
& Self-reported diary & One work shift & Contact persons \\
Doctors and assorted HCWs & Direct observation & $2-8 \mathrm{~h}$ & Contact episodes \\
& Self-reported diary & One work shift & Contact persons \\
Patients & Direct observation & $24 \mathrm{~h}$ & Contact episodes \\
& Self-reported diary & $24 \mathrm{~h}$ & Contact persons \\
Visitors & Telephone interview & One visit to hospital & Aggregated data for the \\
& & & entire visit by contact classes \\
\hline \hline
\end{tabular}

HCWs, healthcare workers.

contact episode: age (or approximate age range), gender, class of the contact person, episode duration in minutes and whether physical contact occurred. Each contact episode occupied one row of the form.

Telephone interview for visitors: Using a structured questionnaire, each participant was asked by telephone for the total duration of that specific hospital visit and for each of the contact classes, the aggregate number of all contact persons and contact persons with whom physical contact occurred.

\section{Data analysis}

For HCWs and patients, the characteristics of their contacts at the participant level were analyzed from four perspectives: the self-reported number of contact persons and from direct observation, the number of contact episodes, the cumulative contact time and median duration of individual contact episodes. We present these measures for each participant type, with further stratification by the class of the person contacted (three classes of HCWs, patients, visitors). To account for differences in the period of assessment for nurses and non-nursing-HCWs (doctors and assorted HCWs), direct observation data for all HCW types were pro-rated to an hourly basis when making any comparisons. All analyses were performed for total contact persons and episodes and then repeated for contact persons and episodes where physical touch occurred. We also summarized the interaction between different classes of persons in contact with each of the participant types using modified contact matrices. The matrices give the proportion of all contacts for that participant type (nurses, doctors, assorted HCWs and patients) and provide a simple representation of the potential risk of exposure for different classes of contact persons should an individual belonging to that particular participant type becomes the source for an infection. This was done for various measures of contact characteristics, selected to depict infections with different transmission characteristics.

Contacts reported by visitor participants during their hospital visit were analyzed separately.

Comparisons were made either using the Wilcoxon rank sum test or the Kruskal-Wallis one-way analysis of variance (for two or more groups, respectively), with two-tailed $P$-values of $<0.05$ considered statistically significant. All analyses were performed using $\mathrm{R}$ version 3.0.2 [13].

\section{RESULTS}

We recruited 377 individuals (Table 2). HCW participants ranged from 20 to 47 years and were predominantly $(81 \cdot 3 \%)$ female. Patients and visitors had a wider age range, with more female visitors $(76 \cdot 6 \%) .65,9,22$ and 40 self-reported diaries, and 57, 16, 35 and 60 direct observation surveys for nurses, doctors, assorted HCWs and patients respectively were available for analysis. We also completed 175 telephone-interview questionnaires for visitors.

Through self-reported diaries, nurses, doctors, assorted HCWs and patients reported a total of 929 , 174, 384 and 576 contact persons respectively, and from direct observation, 2078, 325, 600 and 1410 contact episodes with cumulative durations of 15505 , 2762, 6163 and $25272 \mathrm{~min}$.

\section{Contacts of HCWs (Fig. 1 and supplementary Tables S1-S2)}

Over one work shift, nurses reported a median of 14 (range 3-29), doctors reported 18 (range 12-26) while assorted HCWs reported 15 (range 3-40) contact persons. Within $1 \mathrm{~h}$, we observed 3.5 (range 1.3-15.5) episodes of contact and 25.6 (range 9.2133.4) min of cumulative contact time for nurses; $2 \cdot 9$ 
Table 2. Characteristics of individuals by participant types

\begin{tabular}{llllll}
\hline \hline & Nurses & Doctors & Assorted HCWs & Patients & Visitors \\
\hline $\begin{array}{l}\text { Number } \\
\text { Age }\end{array} \quad$ Median (range) & 71 & 17 & 40 & 74 & 175 \\
$\begin{array}{l}\text { Gender } \\
\quad \text { Female (\%) }\end{array}$ & $27(20-47)$ & $26(24-47)$ & $25(22-42)$ & $59(21-88)$ & $42(21-78)$ \\
$\begin{array}{l}\text { Survey method } \\
\quad \text { Self-reported only (\%) }\end{array}$ & $63(88 \cdot 7)$ & $8(47 \cdot 1 \%)$ & $33(82 \cdot 5$ & $31(41 \cdot 9)$ & $134(76 \cdot 6)$ \\
$\quad$ Direct observation only (\%) & $14(19 \cdot 7)$ & $1(5 \cdot 9 \%)$ & $5(12 \cdot 5)$ & $14(18 \cdot 9)$ & - \\
$\quad$ Both (\%) & $51(71 \cdot 8)$ & $8(47 \cdot 1 \%)$ & $18(45 \cdot 0)$ & $34(46 \cdot 0)$ & - \\
$\quad \begin{array}{l}\text { Ward } \\
\quad \text { Non-single-room }\end{array}$ & $66(47 \cdot 1 \%)$ & $17(42 \cdot 5)$ & $26(35 \cdot 1)$ & - \\
$\quad$ Single-room & $5(7 \cdot 0)$ & - & - & $68(91 \cdot 9)$ & - \\
\hline \hline
\end{tabular}

HCWs, healthcare workers.

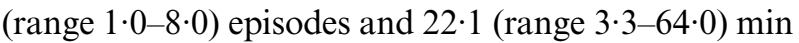
for doctors; and 5.0 (range 1.2-11.0) episodes and $44 \cdot 3$ (range 3.3-161.7) min for assorted HCWs. Doctors reported more patients as contact persons than other HCWs (nurses $v s$. doctors $v s$. assorted HCWs: median of 7 vs. 8 vs. 3 per work shift, Fig. 1a). However, nurses had the most contact episodes with patients (nurses $v s$. doctors $v s$. assorted HCWs: 1.8 vs. $0.8 v s$. 1.3 contact episodes per hour, Fig. $1 b$ ). In contrast, assorted HCWs had the longest cumulative contact time and longest contact episode duration with patients (nurses vs. doctors vs. assorted HCWs: 13.9 vs. 11.4 vs. $17.2 \mathrm{~min}$ of cumulative contact time per hour, Fig. 1c). Interactions between HCWs of the same type (e.g. nurses with nurses) were more frequent and lasted longer than other HCW interactions (Fig. $1 a-c$ ). The duration of HCWs' individual contact episodes were generally short (Fig. 1d, 47.1\% $\leqslant 3 \mathrm{~min}$, and $64.9 \% \leqslant 5 \mathrm{~min}$ ). However, individual contact episodes with patients for assorted HCWs were substantially longer (median of $10 \mathrm{~min}$, vs. 5 min for both nurses and doctors).

When restricted to contact persons with physical touch, both nurses and doctors had the same median number of patients, and substantially more than for assorted HCWs (nurses vs. doctors vs. assorted HCWs: 6 vs. 6 vs. $0 \cdot 5$ per work shift, Fig. 1a). However, based on contact episodes where physical touch occurred, nurses were observed to have the most contact episodes and the longest cumulative contact time with patients, followed by assorted HCWs, with doctors having much fewer episodes and much less time with physical contact (nurses $v s$. doctors $v s$. assorted HCWs: $1 \cdot 6$ vs. $0 \cdot 1$ vs. $0 \cdot 8$ contact episodes per hour, 11.9 vs. $1.3 \mathrm{vs} .9 \cdot 7 \mathrm{~min}$ of cumulative contact time per hour, Fig. 1b-c). Episodes involving physical contact with patients (Fig. 1d) lasted longer than those without for nurses and assorted HCWs while no difference was observed for doctors (nurses: median of 5 vs. 2 min, assorted HCWs: 15 vs. 3 min, $P<0.001$ for both; doctors: 5 vs. $5 \mathrm{~min}, P=0.895$ ).

\section{Contacts of patients (Fig. 2 and supplementary Tables S1-S2)}

Over $24 \mathrm{~h}$, patients had 14 (range 2-40) contact persons and 23 (range 10-44) contact episodes with 314.5 (range 51-1851) min of contact time; for physical contacts, patients had seven (range 0-19) contact persons, 13 (range 2-32) contact episodes and 193 (range 4-1682) min of contact time. Nurses contributed most to patients' contact persons $(6$, range $0-19$, Fig. 2a) and contact episodes (11, range 5-26, Fig. 2b), whereas visitors contributed most to patients' cumulative contact time (167 min, range 0-1821 min, Fig. 2c). In concurrence with direct observations of HCWs, patients' contact episodes with HCWs were short (median of 3, 5 and 5 min for nurses, doctors and assorted HCWs respectively, Fig. 2d), while $65 \cdot 2 \%$ of episodes with visitors lasted $\geqslant 30 \mathrm{~min}$ and $38.8 \% \geqslant 1 \mathrm{~h}$. While contact with other patients was rare (median of 0 ), the maximum number of other patients reported as contact persons was 9, and we documented a maximum of 5 contact episodes with other patients over $24 \mathrm{~h}$ of direct observation (for a different patient). Median contact episode duration with other patients was $10 \mathrm{~min}$, with around $10 \%$ lasting longer than $30 \mathrm{~min}$; episodes where physical contact occurred lasted longer than those without physical contact $(22.5$ vs. $10 \mathrm{~min}, P<0.001)$. 
(a)

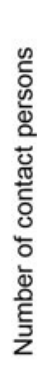

(b)
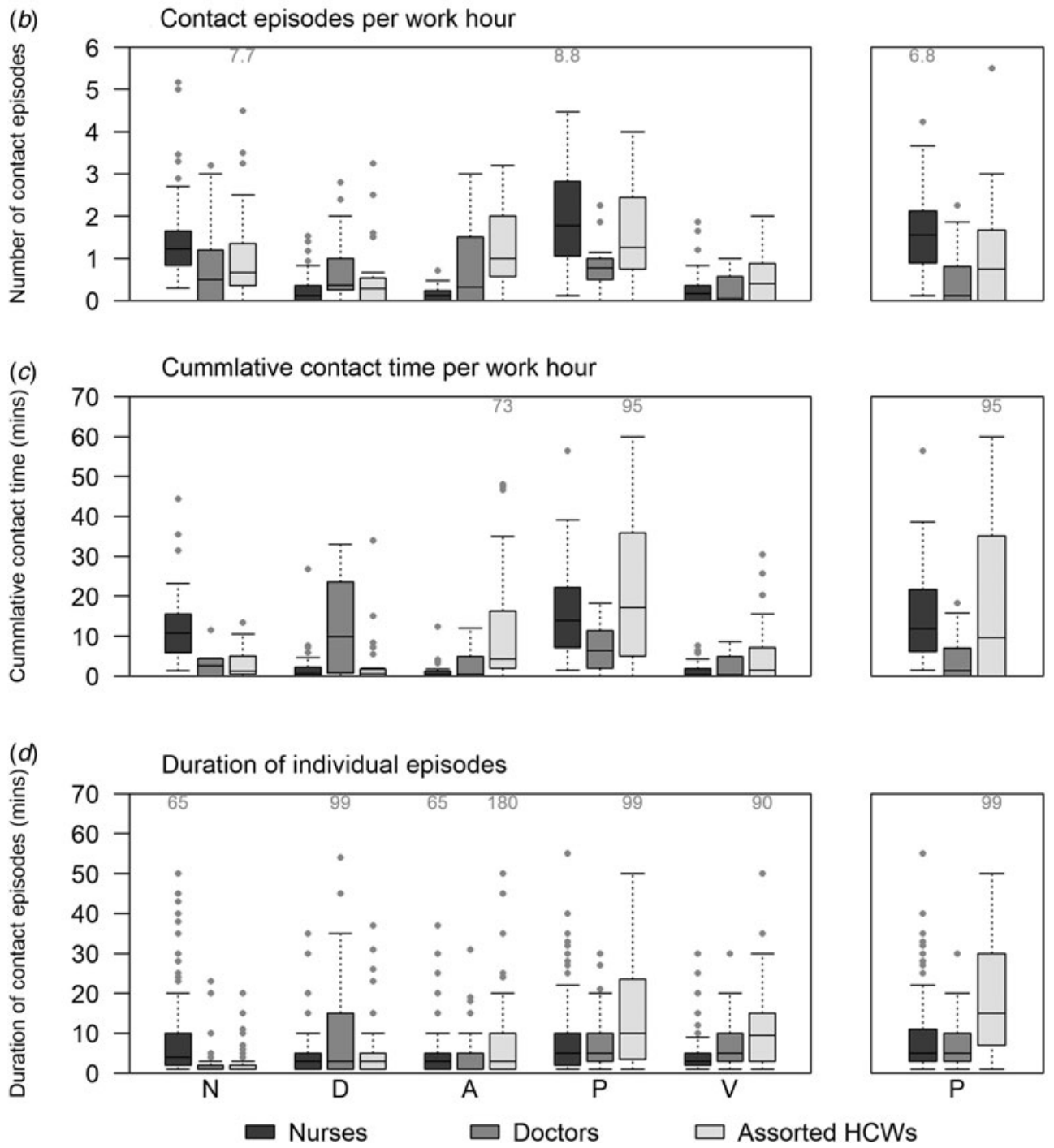

Fig. 1. Summary of contacts for HCWs. The panels represent four measures of contacts: $(a)$ the number of contact persons per work shift, $(b)$ the number of contact episodes per work hour, $(c)$ cumulative contact time per work hour, and (d) duration of individual contact episodes. The left side of each panel includes all contacts, the right side of each panel includes only those contacts with patients where physical contact occurred. Nurses, doctors and assorted HCWs' contacts are stratified (on the horizontal axes) by the class of contact persons $(\mathrm{N}$, nurses; D, doctors; A, assorted HCWs; P, patients; $\mathrm{V}$, visitors). Boxplots represent the distribution (median as a centre line, inter-quartile range (IQR) in the span of the columns, and the upper and lower whiskers represent the highest and lowest values which are no greater than 1.5 times the IQR); for heavy-tailed distributions with a large range compared with the median, the value of the extreme outlier is stated (instead of listing all outliers). 

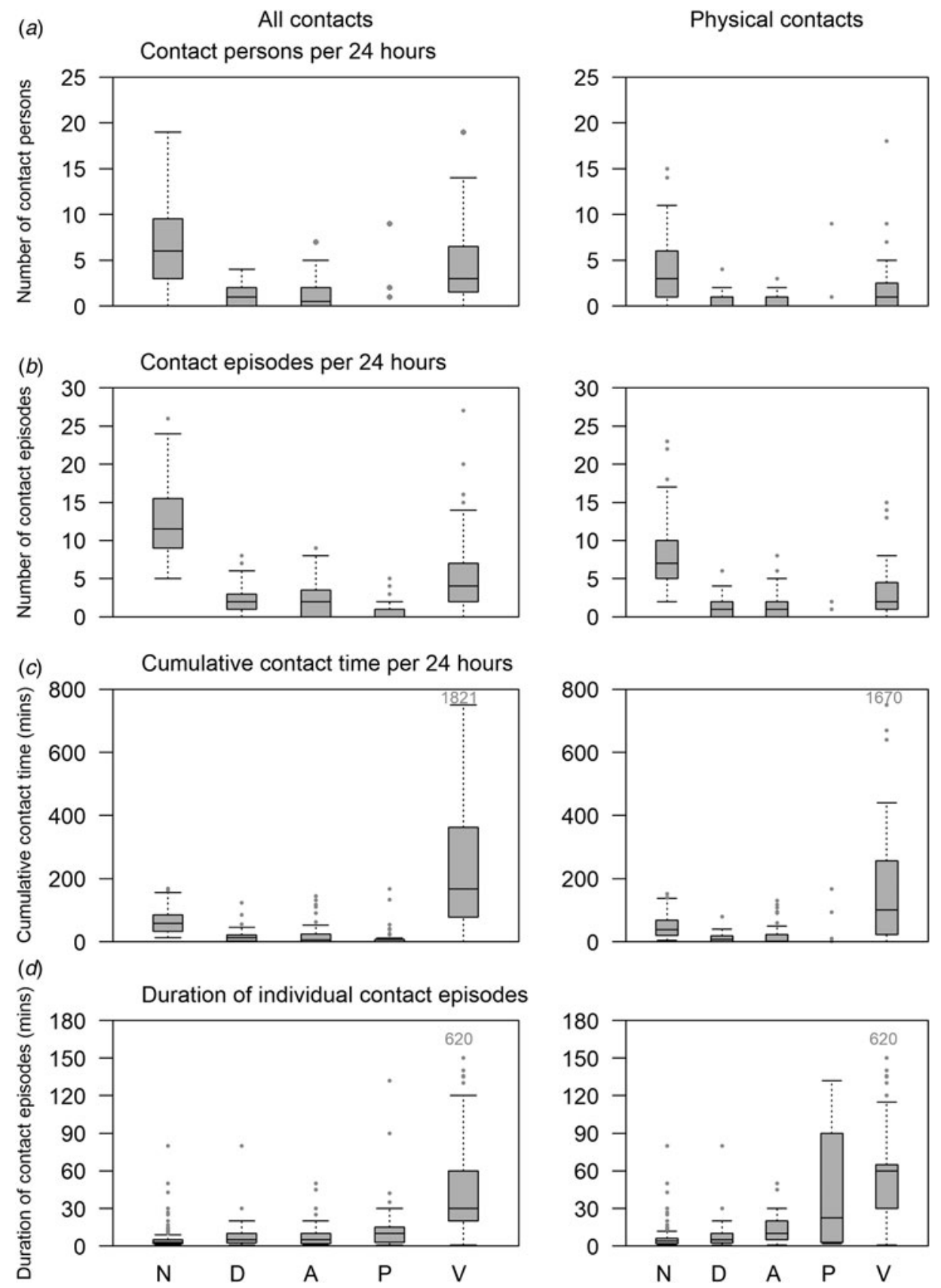

Fig. 2. Summary of contacts for patients. The panels represent four measures of contacts: $(a)$ the number of contact persons per day, $(b)$ the number of contact episodes per day, $(c)$ cumulative contact time per day, and $(d)$ duration of individual contact episodes. The left side of each panel includes all contacts, whereas the right side of each panel includes only those contacts where physical contact occurred. Patients' contacts are stratified (on the horizontal axes) by the class of contact persons (N, nurses; D, doctors; A, assorted HCWs; P, patients; V, visitors). Boxplots represent the distribution (median as a centre line, inter-quartile range in the span of the columns, and the upper and lower whiskers represent the highest and lowest values which are no greater than 1.5 times the IQR); for heavy-tailed distributions with a large range compared to the median, the value of the extreme outlier is stated (instead of listing all outliers).

\section{Modified contact matrices (Fig. 3 and supplementary Table S3)}

Regarding the total number of contact persons (Fig. 3a), from the perspective of the participant type, patients were the most frequent class of contact for HCWs $(56 \%, 41 \%$ and $43 \%$ for nurses, doctors and assorted HCWs, respectively). However, there was assortative mixing amongst HCWs, with the 
(a) Total number of contact persons

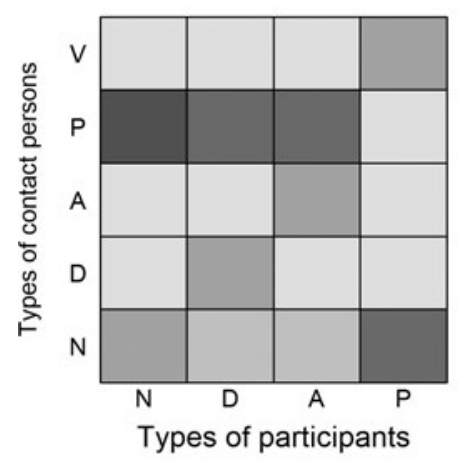

(c) Total number of contact episodes

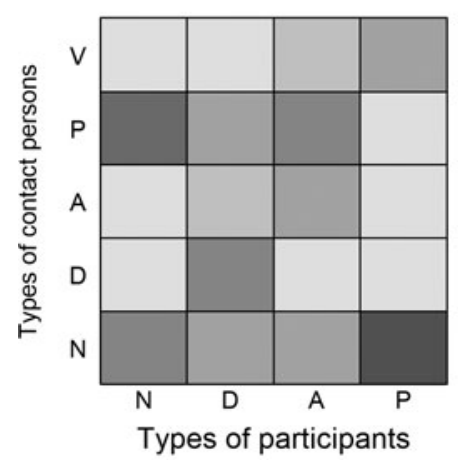

(b) Cumulative contact time observed

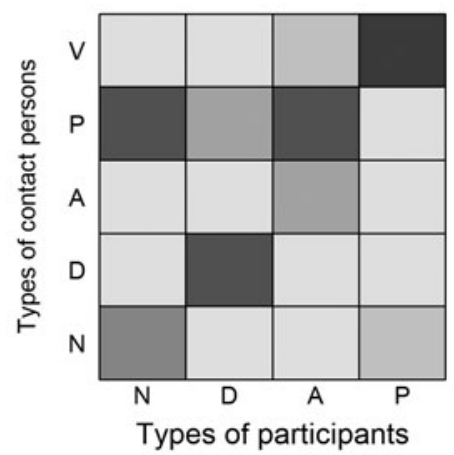

(d) Number of episodes with physical touch

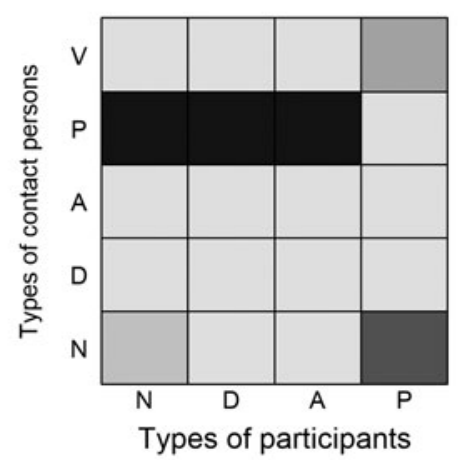

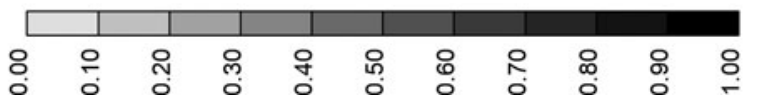

Fig. 3. Contact matrices based on the distribution of: $(a)$ Total number of reported contact persons, $(b)$ cumulative contact time, $(c)$ total number of observed contact episodes, $(d)$ number of episodes with physical touch. Each cell of the matrix represents the proportion of the contacts occurring with a specific class of contact persons for the type of participant represented by that column. Note that the column totals do not add up to $100 \%$ because the observations where the contact class was 'Others/Missing' are excluded from the figure.

next most frequent class of contacts being those of the same $\mathrm{HCW}$ type $(24 \%, 25 \%$ and $22 \%$ for nurses, doctors and assorted HCWs, respectively). From the patients' perspective, nurses contributed most to total contacts $(48 \%)$, followed by visitors $(30 \%)$. However, for cumulative contact time with patients (Fig. 3b), visitors were the main contact class $(69 \%)$, followed by nurses, assorted HCWs and doctors (16\%, 5\% and 4\%, respectively). Amongst the HCWs, cumulative contact time was also partially assortative, particularly in doctors, who spent more time in contact with other doctors $(57 \%)$ than they did with patients (24\%); nurses and assorted HCWs spent most of their contact time with patients $(50 \%$ and $51 \%$, respectively) followed by those of the same HCW type (37\% and 24\%, respectively). However, when analyzing contact episodes (Fig. 3c), for the doctors and assorted HCWs, these showed a fairly even distribution across the contact classes, except that most contact episodes for the doctors were amongst themselves $(32 \%)$, while the class of contacts that assorted HCWs had the least contact with were the doctors $(10 \%)$. For nurses, contact episodes were largely concentrated amongst patients $(48 \%)$ and other nurses $(35 \%)$; from the patients' perspective, $53 \%$ of contact episodes were with nurses, followed by visitors $(23 \%)$. However, for physical contacts, patients contributed more than $80 \%$ of episodes for all three HCW participant types (Fig. 3d). Physical interactions between $\mathrm{HCWs}$ were infrequent; the most frequent such interaction was between nurses ( $12 \%$ of episodes, median of $0 \cdot 17$ episode per hour). From the patient's perspective, the majority of physical contact episodes were with nurses $(58 \%)$, followed by visitors $(22 \%)$, with much smaller proportions being with doctors $(10 \%)$ and assorted HCWs (9\%). 


\section{Effect of work shift, ward type and day-of-week on the contacts of nurses and patients}

Nurses had less contact episodes and contact time with patients during the afternoon as compared with other shifts. On weekends, nurses had more but shorter contacts with patients than on weekdays. Compared with non-single-room wards, patients in single room wards had fewer unique nurse contacts and also fewer contact episodes, but a longer cumulative contact time. Patient-to-patient contacts occurred more frequently in non-single-room wards and on weekdays. However, none of these differences were statistically significant.

\section{Summary of visitors' questionnaire survey}

Among the 175 visitors, $79 \cdot 4 \%$ had physical contacts with the patients they visited; $76.0 \%$ had contacts with HCWs (but only $5 \cdot 7 \%$ involved physical touch), with a median 2 (range $0-12$ ) HCW contacts. $22 \cdot 9 \%$ reported contact with patients other than the one they visited, although physical contact was infrequent (1.7\%). 64.6\% had contact with other visitors (median of 1 visitor); $18.9 \%$ had physical contact with other visitors (Table 3).

\section{DISCUSSION}

Our study provides several measures of contacts among HCWs, patients and visitors within the setting of the acute care general ward in Singapore. In contrast to studies like those by Cohen et al. [6] and Raboud et al. [7], which focused on and recorded all visits to a specific patient's room, we also covered contacts within the ward that were outside the patient's room, with a timeframe for different types of participants scheduled to be in line with their exposure to the ward. Some of this was previously described by others using proximity sensing technologies [10, 14, 15]. However, by using both self-reported diaries with direct observation to quantify the number and duration of all contacts and physical contacts, we showed that the way contacts are measured can have different implications as to the key groups at-risk for acquiring, as well as spreading different types of infection. For instance, nurses had multiple brief episodes of contact with patients that often involved physical touch; doctors, while reporting contact with a similar number of patients, had fewer episodes of contact and consequently less cumulative contact time with patients.
On the other hand, assorted HCWs (which mainly included therapists and other allied health staff) had similar cumulative contact time as nurses, but this arose from fewer episodes of contacts with longer durations than those typically observed in nurses. The HCWs contacts with patients were consistent with the respective HCW's role in patient care, and are relevant to understanding potential pathways for transmission. Measuring these contacts also provides parameters to support modeling of disease transmission in such an environment.

For instance, for drug-resistant bacteria, a significant threat to public health [16], physical patient-to-HCWto-patient contacts has been suspected as a major pathway for transmission [17, 18]. For all HCW types, patients were the most common class of physical contacts. However, on observing the patients, we found that nurses had a far higher number of, and accounted for close to $60 \%$ of all episodes with physical contact compared with $<10 \%$ each for doctors and assorted HCWs (Fig. 3d). If transmission of such bacteria is more dependent on episodes of physical touch rather than the number of patients contacted, then nurses could potentially (in the absence of interventions) play a bigger role in the spread of these pathogens, even though they reported physical contact with the same median number patients as doctors. The possible role of nurses was previously highlighted in other studies, which used proximity-sensing technologies [13, 14] that do not explicitly measure contacts involving physical touch, which we measure here. Previous studies also led others $[9,15]$ to comment that nurses may be a priority for infection control interventions due to the characteristics of their contacts. Our study provides additional support for this and emphasizes the importance of this for drug-resistant bacteria where physical contact is important for transmission [19].

Our findings also have relevance to transmission of respiratory infections like influenza, which has and continues to cause nosocomial outbreaks [20], as well as novel infections like SARS and MERS coronaviruses where a substantial fraction of cases arise from outbreaks in healthcare settings [21]. It is unclear for many of these pathogens what parameters are most critical to transmission. For influenza, we previously observed during the influenza $\mathrm{A}(\mathrm{H} 1 \mathrm{~N} 1) \mathrm{pdm} 09$ pandemic of 2009 that contact with other colleagues, and also patients with confirmed influenza $\mathrm{A}(\mathrm{H} 1 \mathrm{~N} 1)$ pdm09 infections, both independently increased the risk of infection [3]. This suggests the importance of both HCW-to-HCW and patient-to-HCW pathways 
Table 3. Summary of visitors' contacts

\begin{tabular}{|c|c|c|c|c|c|}
\hline Who the visitor contacted: & Patient they visited & HCWs & $\begin{array}{l}\text { Patients besides } \\
\text { the one they visited }\end{array}$ & Other visitors & Others \\
\hline \multicolumn{6}{|l|}{ Total contact } \\
\hline No. $(\%)$ had contact & $175(100)$ & $133(76 \cdot 0)$ & $40(22 \cdot 9)$ & $113(64 \cdot 6)$ & $12(6 \cdot 9)$ \\
\hline Total no. of contact persons & 175 & 399 & 71 & 385 & 14 \\
\hline Median no. of contact persons (range) & $1(1-1)$ & $2(0-12)$ & $0(0-6)$ & $1(0-12)$ & $0(0-3)$ \\
\hline \multicolumn{6}{|l|}{ Physical contact } \\
\hline No. $(\%)$ had contact & $139(79 \cdot 4)$ & $10(5 \cdot 7)$ & $3(1 \cdot 7)$ & $33(18 \cdot 9)$ & $2(1 \cdot 1)$ \\
\hline Total no. of contact persons & 139 & 44 & 7 & 103 & 4 \\
\hline Median no. of contact persons (range) & $1(0-1)$ & $0(0-7)$ & $0(0-3)$ & $0(0-10)$ & $0(0-3)$ \\
\hline
\end{tabular}

HCWs, healthcare workers.

for transmission. If transmission is highly dependent on the number of contact persons or cumulative contact time (where we observed assortative mixing amongst HCWs, Fig. $3 a-b$ ), then the initial infections would most probably be concentrated amongst HCWs of same type after its introduction into a given setting by an infectious $\mathrm{HCW}$. On the other hand, following introduction by an infectious patient, our observations suggest that nurses would be most at-risk, as amongst HCWs, they were proportionately most in contact with patients on all the measures presented in Figure 3. Quantification of the risk to different HCW types, and how an epidemic might evolve in subsequent generations of transmission would require that we use models that integrate information about the numbers of contact persons and episodes with information on contact time. While the theoretical importance of including information on the duration of contacts has been recognized [22, 23], models of influenza transmission in the healthcare setting have thus far focused mainly on the number of contacts $[24,25]$, possibly due to the unavailability of the relevant parameters, which we have now measured.

Our findings may also explain several epidemiological observations associated with the outbreaks of SARS-CoV in 2003. In Vietnam, Canada and Singapore, HCWs accounted for $57 \%, 43 \%$ and $41 \%$ of infections, respectively [26]. In Singapore, most $\mathrm{HCW}$ infections arose from super-spreading events and patient-to-HCW transmission from unrecognized cases of the infection [27]. Our investigation of the patients' contacts confirms that, other than visitors who cumulatively have the most contact time with inpatients, ward-based nurses are the class of contacts most exposed to a potentially infectious case within the general ward environment by all the other measures used. Indeed, during key hospital-based outbreaks in
Singapore, nurses outnumbered other HCWs by about 2:1 [3, 28]. Moreover, visitors to hospitals were another sizeable group that was infected $(\sim 20 \%$ of Singapore's SARS-CoV cases) [27]. The contacts of visitors has largely been neglected in other studies in hospital settings [7, 8, 10, 29], possibly due to the challenges in collecting such information. Direct observation of patients showed that contact episodes with visitors were often prolonged, and our telephone questionnaire suggests that almost $80 \%$ of visitors had physical contact with the patients they visited. Moreover, $20 \%$ had contacts with patients besides the one they visited, which could explain why a substantial number of visitors to non-SARS-CoV patients were infected [30]. As for patient-to-patient transmission, which occurred less frequently with SARS-CoV but has featured prominently in MERS-CoV outbreaks [21], we note that while patient-to-patient contacts were relatively rare, physical and prolonged contacts do occur. This pathway may hence deserve greater attention for interventions to interrupt nosocomial transmission. Finally, the role of super-spreaders has been highlighted in several studies of SARS-CoV [31], MERS-CoV [32], and even Ebola virus outbreaks [33]. A large number of contacts have been suspected as a possible contributory mechanism $[34,35]$. Amongst our inpatients, the distribution of contact persons was moderately right-skewed, with a median of 14 contact persons but a maximum of 40 over a $24-\mathrm{h}$ period. Our results could potentially be used to model the extent to which the variation in the characteristics of their contacts contributes to super-spreader phenomena.

Our study has several limitations. Firstly, our participants might not represent typical hospital constituents. Doctors were inadequately represented, and the assorted HCW group aggregated a diversity of healthcare staff types. Patients in our study had to be able to 
give consent for participation, and thus may be younger, have less severe disease and be more functionally mobile than the average inpatient. Also, most visitor participants were recruited from non-single-room wards due to difficulties in approaching visitors from single room wards; the conclusion from the visitors' survey may thus be valid only for non-single room wards. The self-reported survey may also have underestimated the total number of contacts, especially contacts of shorter duration due to recall bias or incomplete recording of contacts [36], and the Hawthorne effect could have biased the results from direct observation. Finally, we acknowledge that beyond what we can infer from our description of contacts in this paper, a more complete understanding of transmission pathways and potential interventions would require that we integrate such data in a modelling approach and validate the outputs against the complex patterns observed in reports of nosocomial outbreaks.

In conclusion, the various measures of contacts in our study revealed subtle but important differences between different types of HCWs, while observations in patients and visitors also highlighted several potential transmission routes within the ward environment. These findings have implications for understanding transmission and designing interventions, and can be used as input parameters for modelling the spread of various healthcare associated infections and how to prevent them.

\section{SUPPLEMENTARY MATERIAL}

The supplementary material for this article can be found at https://doi.org/10.1017/S0950268817002035.

\section{ACKNOWLEDGEMENTS}

We would like to acknowledge TTSH Nursing Service and the participating wards for supporting our study. This work was funded by the National Medical Research Council of Singapore (NMRC/CSA/011/2009).

\section{DECLARATION OF INTEREST}

All other authors report no conflicts of interest related to this article.

\section{REFERENCES}

1. Bernard H, et al. Nurses' contacts and potential for infectious disease transmission. Emerging Infectious Diseases 2009; 15: 1438-1444.
2. Pollara CP, et al. Nosocomial outbreak of the pandemic influenza A (H1N1) 2009 in critical hematologic patients during seasonal influenza 2010-2011: detection of oseltamivir resistant variant viruses. BMC Infectious Diseases 2013; 13: 127.

3. Chen MIC, et al. The outbreak of SARS at Tan Tock Seng Hospital--relating epidemiology to control. Annals of the Academy of Medicine, Singapore 2006; 35: 317-325.

4. Stefani S, et al. Meticillin-resistant Staphylococcus aureus (MRSA): global epidemiology and harmonisation of typing methods. International Journal of Antimicrobial Agents 2012; 39: 273-282.

5. Collins AS. Preventing health care-associated infections. In: Hughes RG, ed. Patient Safety and Quality: An Evidence-Based Handbook for Nurses. Rockville, MD: Agency for Healthcare Research and Quality (US), 2008; 1102-1125.

6. Cohen B, et al. Frequency of patient contact with health care personnel and visitors: implications for infection prevention. Joint Commission Journal on Quality and Patient Safety / Joint Commission Resources 2012; 38: 560-565.

7. Raboud $\mathbf{J}$, et al. Patterns of handwashing behavior and visits to patients on a general medical ward of healthcare workers. Infection Control and Hospital Epidemiology 2004; 25: 198-202.

8. Lowery-North DW, et al. Measuring social contacts in the emergency department. PLoS ONE 2013; 8: e70854.

9. Mastrandrea R, Fournet J, Barrat A. Contact patterns in a high school: a comparison between data collected using wearable sensors, contact diaries and friendship surveys. PLoS ONE 2015; 10: e0136497.

10. Vanhems $\mathbf{P}$, et al. Estimating potential infection transmission routes in hospital wards using wearable proximity sensors. PLoS ONE 2013; 8: e73970.

11. Mikolajczyk RT, Kretzschmar M. Collecting social contact data in the context of disease transmission: prospective and retrospective study designs. Social Networks 2008; 30: $127-135$.

12. Mossong $\mathbf{J}$, et al. Social contacts and mixing patterns relevant to the spread of infectious diseases. PLoS Medicine 2008; 5: e74. doi:10.1371/journal.pmed.0050074.

13. R Core Team. $R$ : A Language and Environment for Statistical Computing. Vienna, Austria: R Foundation for Statistical Computing, 2013.

14. Gundlapalli A, et al. Social network analyses of patienthealthcare worker interactions: implications for disease transmission. AMIA Annual Symposium Proceedings 2009; 2009: 213-217.

15. Isella $\mathbf{L}$, et al. Close encounters in a pediatric ward: measuring face-to-face proximity and mixing patterns with wearable sensors. PLoS ONE 2011; 6: e17144.

16. Friedman ND, Temkin E, Carmeli Y. The negative impact of antibiotic resistance. Clinical Microbiology and Infection 2016; 22: 416-422.

17. Huttunen R, Syrjänen J. Healthcare workers as vectors of infectious diseases. European Journal of Clinical Microbiology \& Infectious Diseases 2014; 33: 1477 1488. 
18. Albrich WC, Harbarth S. Health-care workers: source, vector, or victim of MRSA? The Lancet. Infectious Diseases 2008; 8: 289-301.

19. Bassetti M, et al. Preventive and therapeutic strategies in critically ill patients with highly resistant bacteria. Intensive Care Medicine 2015; 41: 776-795.

20. Salgado CD, et al. Influenza in the acute hospital setting. The Lancet Infectious Diseases 2002; 2: 145-155.

21. Chowell G, et al. Transmission characteristics of MERS and SARS in the healthcare setting: a comparative study. BMC Medicine 2015; 13: 210.

22. De Cao E, et al. The relative importance of frequency of contacts and duration of exposure for the spread of directly transmitted infections. Biostatistics (Oxford, England) 2014; 15: 470-483.

23. Smieszek T. A mechanistic model of infection: why duration and intensity of contacts should be included in models of disease spread. Theoretical Biology \& Medical Modelling 2009; 6: 25.

24. Ong BS, et al. An individual-based model of influenza in nosocomial environments. In: Bubak M, et al., eds. Computational Science - ICCS 2008. Springer: Berlin, Heidelberg, 2008, 590-599.

25. Polgreen PM, et al. Prioritizing healthcare worker vaccinations on the basis of social network analysis. Infection Control and Hospital Epidemiology 2010; 31: 893-900.

26. WHO. WHO|Summary of probable SARS cases with onset of illness from 1 November 2002 to 31 July 2003. (http://www.who.int/csr/sars/country/table2004_04_21/ en/). Accessed 23 February 2017.

27. Goh K-T, et al. Epidemiology and control of SARS in Singapore. Annals of the Academy of Medicine, Singapore 2006; 35: 301-316.
28. Gopalakrishna G, et al. SARS transmission and hospital containment. Emerging Infectious Diseases 2004; 10: 395-400.

29. Lucet J-C, et al. Electronic sensors for assessing interactions between healthcare workers and patients under airborne precautions. PLoS ONE 2012; 7: e37893.

30. Chow KY, et al. Outbreak of severe acute respiratory syndrome in a tertiary hospital in Singapore, linked to an index patient with atypical presentation: epidemiological study. BMJ (Clinical research ed.) 2004; 328: 195.

31. Chen MIC, et al. Understanding the super-spreading events of SARS in Singapore. Annals of the Academy of Medicine, Singapore 2006; 35: 390-394.

32. Park SH, et al. Outbreaks of Middle East respiratory syndrome in two hospitals initiated by a single patient in Daejeon, South Korea. Infection \& Chemotherapy 2016; 48: 99-107.

33. Lau MSY, et al. Spatial and temporal dynamics of superspreading events in the 2014-2015 West Africa Ebola epidemic. Proceedings of the National Academy of Sciences USA 2017; 114: 2337-2342.

34. Khan AS, et al. The reemergence of Ebola hemorrhagic fever, Democratic Republic of the Congo, 1995. Commission de Lutte contre les Epidémies à Kikwit. The Journal of Infectious Diseases 1999; 179(Suppl. 1): S76-S86.

35. Li Y, et al. Predicting super spreading events during the 2003 severe acute respiratory syndrome epidemics in Hong Kong and Singapore. American Journal of Epidemiology 2004; 160: 719-728.

36. Smieszek T, et al. How should social mixing be measured: comparing web-based survey and sensor-based methods. BMC Infectious Diseases 2014; 14: 136. 